\title{
Complications and number of follow-up visits after using septal stapler in septoplasty*
}

\author{
S. Sainio ${ }^{1}$, K. Blomgren ${ }^{1,2}$, M. Lundberg ${ }^{1,2}$ \\ ' Department of Otorhinolaryngology, University of Helsinki, Helsinki, Finland \\ ${ }^{2}$ Department of Otorhinolaryngology - Head and Neck Surgery, Helsinki University Hospital, Helsinki, Finland
}

Rhinology 57: 4, $273-278,2019$

https://doi.org/10.4193/Rhin18.142

*Received for publication:

July 25, 2018

Accepted: March 13, 2019

\begin{abstract}
Background: Septoplasties have traditionally been closed with transseptal sutures, silicone splints, or packing with nasal tamponade. In 2015, our clinic began to employ a septal stapler. The stapler adheres the mucosa to the septal cartilage with bioresorbable staples, replacing both sutures and silicone splints and limiting the use of nasal tamponade for bleeding cases.

The complications of stapler versus other methods have not been reported on previously. Thus, the aim of this study was to investigate whether the use of stapler in septoplasties makes a difference in complication rates, operation time, or number of follow-up visits when compared to the traditional closure or filling methods.
\end{abstract}

Methodology: Patient records from 101 septoplasties in which the stapler had been used, and a reference group of 356 septoplasties in which the stapler had not been used, were retrospectively reviewed and analysed.

Results: No significant difference was seen in the complication rate between the stapler and the control group. Overall follow-up visits were fewer in the stapler group when compared to the control group, however there was no significant difference in the number of unplanned follow-up visits between the groups.

Conclusions: By using the stapler in septoplasty, the number of postoperative follow-up visits might be reduced. Neither complication rate, nor operation time differed when using the stapler as compared to the traditional methods of closure.

Key words: nasal septum, postoperative complications, septal stapler, septoplasty, suture techniques

\section{Introduction}

Septoplasty is one of the most common operations performed by otorhinolaryngologists ${ }^{(1)}$. At the Department of Otorhinolaryngology - Head and Neck Surgery, Helsinki University Hospital, Finland, septoplasty is the sixth most common surgery, performed an average of 134 times a year. For such a common operation, even small changes in operation time, follow-up visits, and complication rate make a difference.

The complication rate in septoplasties is low. According to a systematic review by Quinn et al. (2013), rates of septoplasty complications are $2.1 \%$ for perforation, $0.7 \%$ for haematoma, and $3.8 \%$ for adhesions ${ }^{(2)}$. Minor haemorrhage has been reported to occur after as much as $34.7 \%$ of septoplasties ${ }^{(1)}$. In 2015, van Egmond et al. reported that complications like nasal obstruction, septal haematoma, septal abscess, nasal deformity, septal perforation, epistaxis, and rhinitis occur in even less than $1 \%$ of cases $^{(3)}$.

One critical step that reduces the complication risk, is minimising the space between the septal layers when finishing the operation ${ }^{(4)}$. Traditionally, this has been done with sutures, silicone splints, or nasal tamponade. These methods contribute to haemostasis and prevent displacement of the remaining septum $^{(4,5)}$.

There are several suturing techniques described for coaptating the mucosa. Whilst suturing is effective, it has been described as time consuming ${ }^{(5)}$. Complications reported after suturing include septal perforations and synechiae formation ${ }^{(5,6)}$. Nasal tamponade or packing is used to prevent complications 


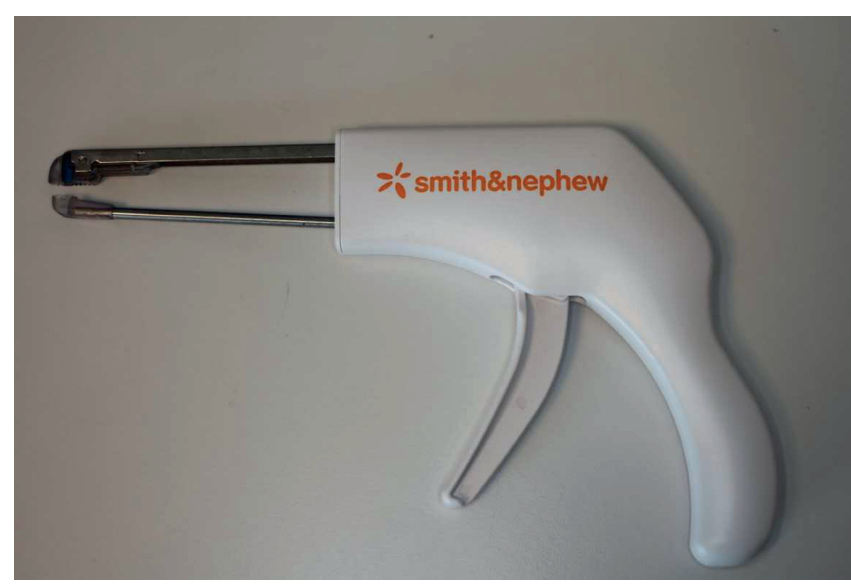

Figure 1. The stapler.

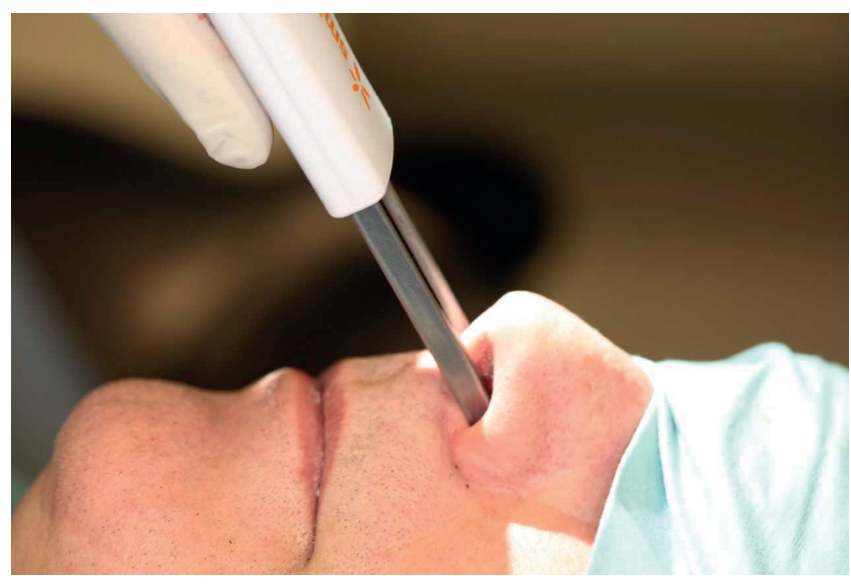

Figure 2. Stapler in use during surgery. such as haemorrhage, haematoma, infection, synechiae formation, and perforation ${ }^{(4)}$. Tamponade can cause great discomfort with reduced nasal breathing, mouth dryness, and nasal pain ${ }^{(7)}$. Patients with postoperative tamponade suffer from more pain than patients with septal sutures, especially removing the packs can cause remarkable pain ${ }^{(7)}$. Other possible complications of tamponade include hypoxia, nasal injury, continued bleeding, foreign-object reaction, and infection. Due to possible complications of tamponade, it has been reported to prolong the hospital stay after septoplasty ${ }^{(7,8)}$.

Septal silicone splints support the nasal septum effectively and prevent adhesion formation ${ }^{(9)}$. Potential complications include foreign-object reaction, infection, and valvular wounds $(9,10)$. Additionally, removal of the splints requires a minimum of one follow-up visit ${ }^{(9,10)}$.

In 2015, a septal stapler (ENTact, Smith \& Nephew, London, UK) was acquired by Helsinki University Hospital, Department of Otolaryngology and Head and Neck Surgery (Figures 1 and 2). The stapler adheres the mucosa to the septal cartilage with bioresorbable staples made of polylactid-co-glycolid (PLG), replacing both sutures and silicone splints and limiting the use of nasal tamponade to bleeding cases. The stapler is easy and quick to use, and has been found to be an effective method in coaptating the mucoperichondrial flaps ${ }^{(11)}$. Postoperatively, patients are equally as comfortable as when using sutures ${ }^{(12)}$. No difference in operation result has been found between the stapler and other methods ${ }^{(12)}$.

The complications of stapler versus other methods have not been studied. As staples are foreign objects, they carry a risk of infection, inflammation, and foreign-object reaction. The pressure from the staples is unevenly distributed over the septum, which may increase the risk of haematoma. Like trans-septal sutures, the staples penetrate the septum, which may enhance the risk of septal perforation.

Of the surgical methods used, the stapler is the most expen- sive. In 2017 the cost per operation was $€ 150.00$ for the stapler; $€ 18.66$ for silicone splints; $€ 13.40$ for two nasal tampons; and $€ 3.60-4.50$ for sutures.

The aim of this study was to investigate whether the use of the stapler in septoplasties makes a difference in complication rate, operation time, or number of follow-up visits when compared to the traditional closure or filling methods.

\section{Materials and methods}

We retrospectively reviewed the 2015 and 2016 patient records from all septoplasties in which a stapler had been used. The control group consisted of all other patients than the patients in the stapler group, that is, 356 patients whose septoplasty was closed with some other method than the stapler. Thus, the control group includes septoplasties closed with silicon splints, tamponade, and sutures. Silicon splints, tamponade, and sutures were used somewhat overlappingly in some cases, that is, tamponade was used in addition to silicon splints or sutures. To avoid bias that could have been caused by the choice of closing certain types of septoplasties with stapler, we also included septoplasties from 2014 (done by the rhinologist that introduced the stapler, and did the majority of the stapler surgeries in 2015).

Since the study was retrospective, and the use of the stapler was new to the clinic, there was no exact scheme neither for the closing method, operations nor the follow-up. Because of the retrospectivity, the method of septoplasty closure was not randomised, and thus depended on the surgeon's choice. The follow-up was individually planned for each patient by the operating surgeon. If there were planned follow-up visits they were scheduled 1-12 weeks postoperatively. The complications included in the study are those noted at short-term (1-12 weeks) routine follow-up or when the patient contacted the emergency room or outpatient clinic. Patient characteristics, smoking, ASAclassifications, use of antibiotics, methods of closure, operation time, complications, and number of follow-up visits were recor- 
Table 1. Number of operations (stapler vs. control) in comparison to the surgeons' experience. Silicone splints, sutures and tamponade are subgroups of the control group, and overlap to some extent.

\begin{tabular}{lcccc} 
& $\begin{array}{c}\text { Stapler } \\
\mathbf{n}(\%)\end{array}$ & $\begin{array}{c}\text { Control, } \\
\text { total } \mathbf{n}(\%)\end{array}$ & $\begin{array}{c}\text { Silicone splints } \\
\mathbf{n}\end{array}$ & $\begin{array}{c}\text { Sutures } \\
\mathbf{n}\end{array}$ \\
\hline Rhinologists & $37(21.3)$ & $137(78.7)$ & 107 & 18 \\
ENT-specialists & $48(21.5)$ & $175(78.5)$ & 93 & 35 \\
\hline Residents & $16(26.7)$ & $44(73.3)$ & 37 & 2
\end{tabular}

Table 2. Number of complications in comparison to pre-operative risk factors and use of antibiotics.

\begin{tabular}{|c|c|c|c|c|}
\hline & & $\begin{array}{c}\text { Total number } \\
\text { n (\%) }\end{array}$ & $\begin{array}{c}\text { Complications } \\
\text { n (\%) }\end{array}$ & p-value \\
\hline \multirow[t]{2}{*}{ Gender } & Men & $349(76.4)$ & $27(7.7)$ & 0.05 \\
\hline & Women & $108(23.6)$ & $15(13.9)$ & \\
\hline \multirow[t]{4}{*}{ Smoking status } & Smoker & $100(21.9)$ & $5(5.0)$ & 0.22 \\
\hline & Ex-smoker & $77(16.8)$ & $10(13.0)$ & \\
\hline & Non-smoker & $148(32.4)$ & $12(8.1)$ & \\
\hline & Unknown & $132(28.9)$ & $15(11.4)$ & \\
\hline \multirow[t]{4}{*}{ ASA-classification } & ASA 1 & 237 (51.9) & $25(20.5)$ & 0.74 \\
\hline & ASA 2 & $181(39.6)$ & $14(7.7)$ & \\
\hline & ASA 3 & 37 (39.6) & $3(8.1)$ & \\
\hline & ASA 4 & $2(0.4)$ & 0 & \\
\hline \multirow[t]{2}{*}{ Previous septal surgery } & Yes & $19(4.2)$ & $3(15.8)$ & 0.57 \\
\hline & No & $438(95.8)$ & $39(9.8)$ & \\
\hline \multirow[t]{2}{*}{ Preoperative antibiotics } & Yes & $195(42.7)$ & $20(10.3)$ & 0.50 \\
\hline & No & $262(57.3)$ & $22(8.4)$ & \\
\hline \multirow[t]{2}{*}{ Postoperative antibiotics } & Yes & $96(21.0)$ & $10(10.4)$ & 0.64 \\
\hline & No & $361(79.0)$ & $32(8.9)$ & \\
\hline
\end{tabular}

ded and analysed.

Altogether 49 surgeons' operations were reviewed, and of these surgeons, 22 used the stapler. Some of the surgeons performed operations both with and without stapler, while others performed operations solely on the stapler or the reference group. We classified the surgeons based on their experience as rhinologists, ENT-doctors, and residents. Of the stapler operations, the rhinologists performed $36.6 \%$, ENT-doctors $47.5 \%$, and residents $15.8 \%$; while the same numbers in the control operations were $38.6 \%, 49.3 \%$, and $12.1 \%$ (Table 1 ).

\section{Statistics}

The material was analysed using NCSS 8 (Hintze, J. \{2012\}; NCSS 8. NCSS, LLC. Kaysville, UT, USA. www.ncss.com), applying the functions Analysis of Variance, Cross Tabulation, and Two-Sample T-Test. A p-value $<0.05$ was considered statistically significant.

We also calculated a power analysis based on a large recent study by Dabrowska-Bien et al. with a complication rate of $3.42 \%{ }^{(13)}$. To demonstrate a $20 \%$ decrease in complication rate at the significance level of 0.05 and with power of $90 \%$, a sample of 13,398 patients in both groups would have been needed.

\section{Ethical consideration}

The data is based on patient records and therefore no approval of the ethics committee was needed. Our study has the approval of Helsinki University Hospital Operative profit centre.

\section{Results}

\section{Population}

The material consisted of 457 septoplasties, of which $101 \mathrm{em}$ ployed a stapler.

Age, sex, smoking status, ASA-classification, or the use of prophylactic antibiotics had no effect on complication rate (Table 2 ).

\section{Procedure}

Complication rates, operation time, and the need for follow-up visits for stapler versus the control group are presented in Table 3. Of the subgroups within the control group, nasal tamponade was the most frequently used method and was employed in 
Table 3. Number of operations, complication rate, operation time and follow-up visits.

\begin{tabular}{|lccc|}
\hline $\begin{array}{c}\text { Stapler } \\
\mathbf{n}(\%)\end{array}$ & $\begin{array}{c}\text { Control } \\
\mathbf{n}(\%)\end{array}$ & p-value \\
\hline Operations & $101(22.1)$ & $356(77.9)$ & - \\
\hline Complications & $10(9.9)$ & $32(9.0)$ & 0.80 \\
\hline $\begin{array}{l}\text { Operation time (min) } \\
\text { Planned follow-up visit }\end{array}$ & 63.9 & 64.3 & 0.31 \\
\hline $\begin{array}{l}\text { Unplanned follow-up } \\
\text { visits }\end{array}$ & $17(16.8)$ & $43(12.1)$ & 0.42 \\
$\begin{array}{l}\text { Follow-up visits per } \\
\text { patient, total }\end{array}$ & 0.98 & 1.51 & $<0.001$ \\
\hline
\end{tabular}

Table 4. Complications in 457 patients divided by operation method. One patient in the control group had both infection and adhesions.

\begin{tabular}{lccc}
$\begin{array}{l}\text { Method/ } \\
\text { Complication }\end{array}$ & $\begin{array}{c}\text { Stapler } \\
\mathbf{n}(\%)\end{array}$ & $\begin{array}{c}\text { Control } \\
\mathbf{n}(\%)\end{array}$ & p-value \\
\hline Infection & $6(5.9)$ & $18(5.1)$ & 0.73 \\
Haematoma & $1(1.0)$ & 0 & 0.06 \\
Bleeding & $1(1.0)$ & $9(2.5)$ & 0.35 \\
Perforation & $1(1.0)$ & 0 & 0.06 \\
\hline Synechiae & 0 & $1(0.3)$ & 0.59 \\
Sinusitis & 0 & $4(1.1)$ & 0.29 \\
Fever & $1(1.0)$ & $1(0.3)$ & 0.34 \\
In total & $10(9.9)$ & $32(9.0)$ & 0.80
\end{tabular}

$60.0 \%$ of the operations. In 63 operations (13.8\%), the tamponade was used alone, and in others it was combined with silicone splints, sutures, or stapler (Table 3).

\section{Complications}

As expected, a minority of the patients $(42,9.2 \%)$ experienced short-term postoperative complications within 12 weeks. The most common complications were infection (27 patients, $5.9 \%$ ) and bleeding (10 patients, 2.2\%). Haematoma and septal perforation afflicted one patient each, both of which were from the stapler group. The complications were treated according to their nature, with $95.2 \%$ treated in the outpatient department. One patient in the stapler group required a reoperation because of septal perforation, while one patient in the control group required a reoperation because of adhesion formation.

No significant difference could be seen in complication rate between the stapler and the control groups (Table 4). In the stapler group, 10 patients (9.9\%) experienced some postoperative complication, compared to 32 patients $(9.0 \%)$ in the control group (Table 3). In the subgroups, 23 patients (9.7\%) had a complication in the silicone splint group, 7 patients (12.7\%) had a complication in the suture group, and 26 patients (9.5\%) had a complication in the tamponade group.

\section{Postoperative observation}

Postoperative observation of the patients varied, as some patients had planned follow-up visits whereas others did not. Most patients (410 patients, $89.7 \%$ ) returned for one or more followup visit/s. One patient had a follow-up call with the surgeon and 46 patients (10.1\%) had no follow-up contacts.

Patients in the stapler group had $0.8( \pm 0.7)$ planned follow-up visits on average, while patients in the control group had 1.4 $( \pm 0.7)$ planned follow-up visits.

Altogether 60 patients had to return for an unplanned follow-up visit. Reasons for an unplanned follow-up visit included bleeding, infection symptoms, obstruction, pain, dry mucosa, and problems with the silicone splints or the tamponade.

When summarising planned and unplanned follow-up visits, patients in the stapler group had significantly lower frequency of follow-up visits (0.98) compared to the control group with an average of 1.51 follow-up visits per patient ( $p<0.001$, Table 2 ).

\section{Discussion}

There was no difference in the complication rate between the stapler and the control group. The percentage of infections was smallest in the stapler group, but the difference was not significant. Perforation and haematoma were seen only in the stapler group, with a low rate of $1 \%$ each. Therefore, this study agrees with the small earlier study by Tami et al. (2010) showing that using the stapler is safe ${ }^{(11)}$.

The number of follow-up visits after using the stapler has not been studied previously. We showed patients operated with the stapler required fewer follow-up visits. While stapler use was associated with more unplanned follow-up visits, the number of planned visits was much lower in the stapler group, with a mean of 0.8 visits. Nowadays, this number is probably much lower in our hospital, since experienced surgeons have no need to schedule follow-up visits for their own learning and feedback. Residents and junior consultants do see their patients at follow-up visits, but it is our custom that experienced rhinologists do not schedule a routine follow-up visit after a basic septoplasty. This does not mean the patients are never seen again - if the patient is not scheduled a routine follow-up, the symptom threshold to contact the clinic again is emphasised to him/her should few or low-level symptoms occur.

Because the staples are made of bioresorbable material, there is no need to plan a follow-up visit for extracting foreign material from the nose. The material for this study includes the very first cases of stapler at our hospital. Therefore, it is natural that the surgeons planned follow-up visits to gain experience in observing the normal recovery period after a new method. This study was not yet planned when the stapler was introduced to our 
clinic, thus the number of planned follow-up visits has not been intentionally lowered.

The price of the stapler per operation is higher than the price of other methods of closure. As the number of follow-up visits can be reduced by using the stapler, and as the operation time can be shortened when surgeons get used to the stapler, it is possible that the costs of the stapler per operation can be evened out over a longer time span. Although price is important, this study focused on the safety of using the stapler and economic issues will instead be addressed in future studies.

There are only four previous studies on the use of the stapler, one published in Chinese. Sowerby et al. (2013) reported that the stapler shortens the closure time with a mean of six minutes, however, we observed no significant time saving ${ }^{(5)}$. An important difference between our study and Sowerby's is the size, whilst Sowerby's study included 16 patients, our study included 457 patients. In Sowerby's study, all operations were performed by the same surgeon, while our material consisted of operations performed by different surgeons ${ }^{(5)}$. It is possible that we have a selection bias in the group of surgeons using sutures, as it is mostly one very experienced surgeon who uses suturing at our clinic, whereas the stapler was also used by residents, whose operations naturally take a longer time. It is also possible that the time saving brought by the stapler was not bigger in our study due to the fact that the instrument was new, and using it therefore required some extra time in the beginning. We believe that our study's large and unselected patient material is a strength, making the results generalisable. Our study was retrospective, and the data was collected from patient records. Since earlier studies have been prospective, they have been able to compare patients' situations before and after the operation with, for example, the NOSE scale. In our study, the postoperative observation was unstructured, as some patients had planned follow-up visits, while others had no follow-up visits.

Earlier studies have reported complication rates due to serious complications in even less than $1 \%$, and due to minor haemorrhages in as much as $34.7 \%{ }^{(1-3)}$. Our total complication rate of $9.8 \%$ correlates with earlier results ${ }^{(1-3)}$. Differences in complication rates between studies could be explained by how a compli- cation is defined - in this study, minor deviations from normal healing were also considered as complications. The decision of whether a patient's postoperative condition was a complication and requiring treatment was made by the treating surgeon or doctor on-call, meaning that mild postoperative mucosal reactions might also have been treated as complications with antibiotics. It is possible that post-operative complications have been treated in private hospitals, which would bias the complication rate. This, however, is unlikely, as patients are instructed to contact their own operative unit in case of complications and we are the only clinic within $200 \mathrm{~km}$ providing round-the-clock otorhinolaryngological services. One could argue that a number of complications are not included, as 47 patients were not seen after operation. As patients are instructed to contact the clinic if symptoms occur or persist, we believe all symptomatic complications are included in the material. Nonetheless, it is possible that a perforation or synechiae may have failed to cause symptoms, and therefore these might have been excluded from our complication rate. However, if a so-called complication is asymptomatic and causes the patient no problems, it does not need to be treated, and is therefore irrelevant.

\section{Conclusion}

By using the stapler in septoplasty, the number of postoperative follow-up visits might be reduced. Neither complication rate, nor operation time using the stapler differed from the traditional methods of closure. Therefore, the stapler can safely be used in septoplasties.

\section{Acknowledgements}

The authors wish to thank Timo Pessi, professional statistician, for helping with the statistics in this manuscript.

\section{Authorship contribution}

SS performed the statistical analysis of data. All the authors contributed to the search of data, writing, and reviewing.

\section{Conflict of interest}

None.

\section{References}

1. Eski E, Yilmaz I. Pack free septoplasty: functional outcomes and complications. Kulak Burun Bogaz Ihtis Derg 2015; 25(5): 275-278.

2. Quinn JG, Bonaparte JP, Kilty SJ. Postoperative management in the prevention of complications after septoplasty: a systematic review. Laryngoscope 2013; 123(6): 1328-1333.

3. van Egmond,M M H T., Rovers MM, Hendriks CTM, van Heerbeek N. Septoplasty for nasal obstruction due to a deviated nasal septum in adults: a systematic review. Rhinology
2018; 56(3): 195-208.

4. Ardehali MM, Bastaninejad S. Use of nasal packs and intranasal septal splints following septoplasty. Int J Oral Maxillofac Surg 2009; 38(10): 1022-1024.

5. Sowerby LJ, Wright ED. A comparison of septal stapler to suture closure in septoplasty: a prospective, randomized trial evaluating the effect on operative time. Int Forum Allergy Rhinol 2013; 3(11): 911-914.

6. Sari K, Gul Al, Kantekin Y, Karaaslan O, Gencer ZK. Transseptal Suturing Reduce Patient Anxiety after Septoplasty Compared to Nasal Packing. Acta Medica (Hradec Kralove) 2016; 59(4): 133-136.

7. Mane RS, Patil B, Mohite A. Comparison of septoplasty with and without nasal packing and review of literature. Indian J Otolaryngol Head Neck Surg 2013; 65(Suppl 2): 406-408.

8. Certal V, Silva H, Santos T, Correia A, Carvalho C. Trans-septal suturing technique in septoplasty: a systematic review and meta-analysis. Rhinology 2012; 50(3): 236245.

9. Banglawala SM, Gill M, Sommer DD, Psaltis 
A, Schlosser R, Gupta M. Is nasal packing necessary after septoplasty? A meta-analysis. Int Forum Allergy Rhinol 2013; 3(5): 418-424.

10. Karatas A, Pehlivanoglu F, Salviz M, et al. The effects of the time of intranasal splinting on bacterial colonization, postoperative complications, and patient discomfort after septoplasty operations. Rev Bras Otorrinolaringol (Engl Ed) 2016; 82(6): 654661.

11. Tami TA, Kuppersmith RB, Atkins J. A clinical evaluation of bioresorbable staples for mucoperichondrial flap coaptation in sep- toplasty. Am J Rhinol Allergy 2010; 24(2) 137-139.

12. Yildirim G, Cingi C, Kaya E. Septal stapler use during septum surgery. Eur Arch Otorhinolaryngol 2013; 270(3): 939-943.

13. Dabrowska-Bien J, Skarzynski PH Gwizdalska I, Lazecka K, Skarzynski H. Complications in septoplasty based on a large group of 5639 patients. Eur Arch Otorhinolaryngol 2018; 275(7): 1789-1794.
Sara Sainio

Bachelor of Medicine

Department of Otorhinolaryngology

University of Helsinki

Helsinki

Finland

Tel. +358408323357

E-mail: sara.sainio@helsinki.fi 